OPEN ACCESS

Edited by:

Saskia M. Kelders,

University of Twente, Netherlands

Reviewed by:

Michel Ferrari,

University of Toronto, Canada

Judith Glück,

University of Klagenfurt, Austria

*Correspondence:

Dilip V. Jeste

djeste@health.ucsd.edu

Specialty section:

This article was submitted to

Positive Psychology,

a section of the journal

Frontiers in Psychology

Received: 01 September 2021 Accepted: 16 December 2021

Published: 03 February 2022

Citation:

Treichler EBH, Palmer BW, Wu T-C, Thomas ML, Tu XM, Daly R, Lee EE and Jeste DV (2022) Women and Men Differ in Relative Strengths in Wisdom Profiles: A Study of 659

Adults Across the Lifespan.

Front. Psychol. 12:769294.

doi: 10.3389/fpsyg.2021.769294

\section{Women and Men Differ in Relative Strengths in Wisdom Profiles: A Study of 659 Adults Across the Lifespan}

\author{
Emily B. H. Treichler ${ }^{1,2,3}$, Barton W. Palmer ${ }^{1,2,4}$, Tsung-Chin W ${ }^{3,5}$, Michael L. Thomas ${ }^{6}$, \\ Xin M. Tu ${ }^{3,5}$, Rebecca Daly,2, Ellen E. Lee ${ }^{1,2,4}$ and Dilip V. Jeste ${ }^{1,2,7 *}$ \\ ${ }^{1}$ VA Desert Pacific Mental IIIness Research, Education, and Clinical Center (MIRECC), San Diego, CA, United States, \\ ${ }^{2}$ Department of Psychiatry, University of California, San Diego, San Diego, CA, United States, ${ }^{3}$ Sam and Rose Stein Institute \\ for Research on Aging, University of California, San Diego, San Diego, CA, United States, ${ }^{4}$ VA San Diego Healthcare \\ System, San Diego, CA, United States, ${ }^{5}$ Department of Family Medicine and Public Health, University of California, San \\ Diego, San Diego, CA, United States, ${ }^{6}$ Department of Psychology, Colorado State University, Fort Collins, CO, \\ United States, ${ }^{7}$ Department of Neurosciences, University of California, San Diego, San Diego, CA, United States
}

Wisdom is a multi-component trait that is important for mental health and well-being. In this study, we sought to understand gender differences in relative strengths in wisdom. A total of 659 individuals aged 27-103 years completed surveys including the 3-Dimensional Wisdom Scale (3D-WS) and the San Diego Wisdom Scale (SDWISE). Analyses assessed gender differences in wisdom and gender's moderating effect on the relationship between wisdom and associated constructs including depression, Ioneliness, well-being, optimism, and resilience. Women scored higher on average on the 3D-WS but not on the SD-WISE. Women scored higher on compassion-related domains and on SD-WISE Self-Reflection. Men scored higher on cognitive-related domains and on SD-WISE Emotion Regulation. There was no impact of gender on the relationships between wisdom and associated constructs. Women and men have different relative strengths in wisdom, likely driven by sociocultural and biological factors. Tailoring wisdom interventions to individuals based on their profiles is an important next step.

Keywords: age, positive psychiatry, compassion, self-reflection, emotional regulation

\section{INTRODUCTION}

Wisdom is one of six core virtues shared across cultures (Peterson and Seligman, 2004; Dahlsgaard et al., 2005), and an increasing area of interest in mental health disciplines due to its link with health, mental health, and well-being (Staudinger and Glück, 2011; Webster et al., 2014; Jeste et al., 2020b). Although the study of wisdom was developed and nurtured in the humanities, in recent decades psychology, psychiatry, and related disciplines have begun to study the topic empirically (Bangen et al., 2013; Glück, 2020; Jeste et al., 2020b). Wisdom is a complex, multi-component trait that includes cognitive, reflective, and affective dimensions (Ardelt, 2003). There are several components common across many proposed definitions of wisdom in the literature - viz., pro-social behaviors and attitudes, including empathy and compassion; emotional regulation, with 
a tendency toward stable, positive mood; self-reflection and awareness; ability to acknowledge and tolerate uncertainty and disagreement; decisiveness; and social advising (Meeks and Jeste, 2009). Some experts also consider spirituality to be a component of wisdom, although there is less consensus on the latter conclusion (Jeste et al., 2020c). Therefore, the contemporary empirical model of wisdom includes and integrates pragmatic elements, like effective social and decision-making skills, and broader elements, like acceptance of self and others, that harken back to its philosophical origins.

The burgeoning positive psychiatry subfield focuses on improving outcomes like quality of life and well-being (Jeste et al., 2015), so wisdom has found a natural home there. Similarly to related domains in positive psychiatry such as resilience and optimism, there is evidence that it is possible to increase wisdom, and that doing so also increasing in quality of life and well-being (Kim et al., 2013; McLaughlin et al., 2018; Paredes et al., 2020; Treichler et al., 2020). A recent meta-analysis of randomized controlled trials targeting individual components of wisdom including empathy and compassion found that nearly one-half of the psychosocial or behavioral interventions were effective with medium to large effect sizes (Lee et al., 2020). Therefore, determining differences in levels of wisdom and its components in specific subgroups of people would help target and tailor associated interventions both at the individual and public health level. For example, one study of emerging adults found four distinct wisdom profiles (Booker and Dunsmore, 2016) indicating different levels of need for intervention, and areas of focus for that potential intervention. More broadly, use of wisdom assessment in clinical practice might be useful in identifying which of existing interventions might be the best fit.

In this article, we examine the association between gender and wisdom. Some reviews and theoretical work have pronounced wisdom should be "androgynous" (Aldwin, 2009) and emphasized wisdom constructs must actively avoid gender bias (Levenson, 2009) both in terms of their conceptualization and their measurement. Yet, of the six studies we found in a thorough but non-systematic review of the literature focused on gender differences in wisdom (Ardelt, 2009; Kanwar, 2013; Singh and Dahiya, 2013; Bang, 2015; Cheraghi et al., 2015; Maroof et al., 2015), and the nine additional studies that included secondary or exploratory analyses about gender differences in wisdom (Ardelt, 1997, 2015; Yang, 2001; Webster, 2003; Levenson et al., 2005; Glück et al., 2013; Bang and Zhou, 2014; König and Glück, 2014; Brienza et al., 2018), nine reported gender differences in one or more analysis (Ardelt, 2003, 2009; Webster, 2003; Kanwar, 2013; Singh and Dahiya, 2013; Bang and Zhou, 2014; Cheraghi et al., 2015; Maroof et al., 2015; Brienza et al., 2018). The other six studies did not find gender differences in any analysis.

The direction of the differences identified were mixed. In three studies of the nine studies where gender differences were found, women scored higher on overall wisdom (Webster, 2003; Singh and Dahiya, 2013; Bang and Zhou, 2014). The rest of the studies did not find an overall pattern where one gender scored higher than the other, but rather found differences in one or more subdomains. These subdomain findings as a whole do not create a clear pattern. For example, one study found that women scored higher on the affective or compassionate subscale (Ardelt, 2009) while another found that men scored higher on the same subscale (Maroof et al., 2015). Studies have reported a variety of findings regarding gender differences in the cognitive subdomain, including that women of all ages score lower (Ardelt, 2003), only older women score lower (Cheraghi et al., 2015), and only older men score higher (Ardelt, 2009). Notably, these studies varied in the mean age of the sample, the sample size, the measure(s) of wisdom and well-being used, and the country or region where the data were collected, which also relates to the variability in race and ethnicity of the sample, and the cultural values of the sample. These differences may impact the findings themselves; study found that men in a non-North American scored higher at wise reasoning in conflict than non-North American women, but there were no differences between North American men and women in wise reasoning in conflict, or between women and men in either group for any other subdomain (Brienza et al., 2018). Which conceptualization of wisdom is being measured also plays a role: a study in India using a less frequently used measure found that men scored higher only on the humor subscale (Kanwar, 2013).

Examination of related psychological constructs yields support for gender differences in a subset of wisdom components. A meta-analysis of the 24 character traits making up the six virtues, including wisdom, found differences between women and men in 17 traits, although 13 of these were very small in size (Heintz et al., 2019). There were small to medium effect sizes in appreciation of beauty/excellence, gratitude, kindness, and love, with women scoring higher in each category on average. Evidence for gender differences in emotional regulation is mixed, broadly indicating that women and men utilize different emotional regulation strategies and the choice of strategy impacts mental health outcomes (Nolen-Hoeksema and Aldao, 2011; Nolen-Hoeksema, 2012; Zimmermann and Iwanski, 2014). Women have been consistently reported to have higher empathy and compassion toward others (e.g., Eisenberg and Lennon, 1983; Sprecher and Fehr, 2005; Martins et al., 2013; Pommier et al., 2020). Compassion is the single most important component of wisdom, providing more evidence that wisdom varies by gender.

Gender norms, differential approaches to upbringing and socialization, and other sociocultural factors may support increased development of some areas of wisdom in women compared to men and vice versa. Related work finds that women and men report some variance in terms of how they conceptualize wisdom, where women are somewhat more likely to endorse a "integrative" model while men are somewhat more likely to endorse a "cognitive" model (König and Glück, 2013). This variance may impact the development of wisdom. For example, an international study of 800 female and male adolescents 1518 years old identified different conceptualizations of wisdom; male adolescents tended to describe wise people as calculating, strict, and questioning, while female adolescents tended to conceptualize wise people as cooperative, optimistic, extroverted, and spontaneous (Hollingworth et al., 2013). However, gender differences between adults in wisdom concepts tend to be quite small (e.g., Glück et al., 2009; Glück and Bluck, 2011; König and Glück, 2013), although they do follow the integrative/cognitive 
pattern. Women are more likely to rate confronting mortality and dealing with negative life events as relevant to developing wisdom, while men were more likely to think that studying philosophy was relevant (Glück et al., 2009). Similarly, when women and men with significant work histories are asked to report situations where they have been wise, $44.9 \%$ of men report work experiences, while only $21.2 \%$ of women report work experiences, and are more likely to report a negative experience including a loss or death (Glück et al., 2009). Therefore, although there is notable overlap in wisdom conceptualization across gender, there are also some distinctions. Those distinctions may translate into differences into how people develop or intentionally pursue wisdom (Hollingworth et al., 2013). Ardelt (2003) also suggested that women and men may receive different levels of support from others in the pursuit of different components of wisdom based on gender-based norms; i.e., that an average man might be encouraged to advance their cognitive wisdom through learning and reflection more than an average woman. This idea is supported by evidence that some components of wisdom tend to be societally seen as a "belonging" to one gender, for example, decisiveness is often seen as a masculine trait (Alexander and Andersen, 1993; Ronk, 1993; Hofstede, 1996). This could mean that a woman and a man with baseline equal levels of wisdom, or wisdom capabilities, might have different levels of wisdom cultivation overall, and particularly by subarea due to internal and external factors, both influenced by sociocultural gender norms. Although we expect that sociocultural factors make up the majority of any gender differences in wisdom, there are also biological underpinnings to wisdom (Meeks and Jeste, 2009) which are likely to vary based on different aspects of biological sex (e.g., hormonal variance). Although gender and sex are not the same construct, gender groups show biologically based variances in some ways (Rametti et al., 2011; Hines, 2020; Kiyar et al., 2020).

Thus, the existing work in this area argues for a gender-neutral wisdom construct, but the empirical studies show mixed findings regarding the current gender-based differences in wisdom, which may exist for sociocultural and biological reasons. Identifying and characterizing gender differences is important because it will better illuminate the current wisdom construct as it is being commonly measured today, and whether it differs from the gender-neutral goal originally sought. It also allows for the consideration of individualized pathways to wisdom. The existence of wisdom subdomains, alongside previous discussion regarding individual development and pursuit of wisdom (e.g., Aldwin, 2009; Hollingworth et al., 2013), suggests the existence of 'wisdom profiles,' with varying strengths and weaknesses. It may further suggest that individuals, and perhaps identifiable groups like women and men, may pursue and achieve wisdom and its associated positive psychiatry outcomes, via different paths. Identifying and deeply understanding these groups, profiles, and pathways will facilitate improved understanding of the wisdom concept itself as well as how support development of wisdom and promotion of associated outcomes in positive psychiatry. The growing set of positive psychiatry interventions often target wisdom and its subdomains alongside other related outcomes, like resilience and optimism, and sometimes also intend to improve symptoms, emotional distress, or social disconnectedness (e.g., Lee et al., 2020). This approach may include implicit assumptions that these constructs are linearly associated with each other across individuals and groups. However, given the possibility of "wisdom profiles," that vary among groups like women and men, understanding how those profiles impact the relationship between wisdom and associated constructs targeted in positive psychiatry interventions would be useful for ensuring that these interventions are maximally effective at the group and individual level.

\section{Aims and Hypotheses}

Therefore, in this study, we examined gender differences in a relatively large community-based sample across the adult lifespan using two validated rating scales (Ardelt, 2003; Thomas et al., 2019). We included other relevant measures related to well-being to better understand whether any potential differences in wisdom between women and men impacted the relationship between wisdom and those constructs associated with well-being.

Based on the existing literature, our first hypothesis was that compassion-related domains such as the Affective or Compassionate dimension of the 3-Dimensional Wisdom Scale (3D-WS), and Pro-Social Behaviors and Acceptance of Diverse Perspectives on the San Diego Wisdom Scale would be higher among women. The second hypothesis was that men would score higher on cognitive-related domains like the Cognitive dimension of the 3D-WS and Decisiveness on the San Diego Wisdom Scale. The third hypothesis was that women would score higher on wisdom total scores. Given significant differences between women and men in this same sample in age, income, education, and marital status, these variables were also included in the model. We conducted two sets of exploratory analyses: first, to test whether the magnitude of gender differences in wisdom would vary between more wise and less wise individuals, as posited by Orwoll and Achenbaum (1993) and supported by Ardelt (2009); and second, to test whether gender impacted the relationship between wisdom and measures of well-being, to understand whether potential gender differences might impact how wisdom relates to other constructs typically targeted by positive psychiatry interventions.

\section{MATERIALS AND METHODS}

This study was approved by the University of California, San Diego Human Research Protections Program (\#171635).

\section{Participants}

The participants in this study were recruited from the UCSD Successful Aging Evaluation (SAGE) study, an ongoing project which has been described in previous work (Thomas et al., 2016). Briefly, SAGE is a multicohort study targeting adults across the lifespan. Participants included adults aged 27-103 who are currently living in the community, physically and mentally able to complete the assessments, have access to a phone in their homes, and have no known dementia diagnosis. People living in nursing homes, those who required daily skilled nursing care, and those with a terminal illness were excluded. The study 
oversampled adults over age 75 because adults in this age group tend to be under-represented in studies of aging. Participants were identified through random digit dialing and completed a 25 min initial phone interview followed by a survey that was mailed or completed online. Participants were provided information about the study in a packet to guide them in their decision to participate; however, documented consent was waived by the human protections review board. In this study, we used the assessment done in 2018 or 2019 , when we had data on both the scales of wisdom, creating a cross-sectional dataset of 659 individuals.

\section{Measures}

Two measures of wisdom with good to excellent psychometric properties including reliability, convergent validity, and divergent validity were included in this study. The first was the 39-item 3D-WS, which has three subscales capturing the Cognitive, Reflective, and Affective (Compassionate) dimensions of wisdom (Ardelt, 2003). The second measure was the 24item San Diego Wisdom Scale (SD-WISE) (Thomas et al., 2019) which has six subscales: Social Advising, Decisiveness, Emotional Regulation, Self-Reflection (previously called Insight), Acceptance of Diverse Perspectives (previously called Tolerance for Divergent Values), and Pro-Social Behaviors.

Gender was self-reported with two categorical options: "male" or "female." This approach allowed for examining differences between people identifying as men and women, the two most frequently reported genders (Richards et al., 2016), but has inherent limitations because it does not apply to all people's experience of their gender (Hyde et al., 2019).

Additional measures were included to examine associated constructs: the Center for Epidemiologic Studies Depression Scale (CES-D) (Radloff, 1977), UCLA Loneliness Scale - Third Edition (ULS) (Russell, 1996), The Mental Wellbeing subscale of the SF-36 (Ware, 2000), Life Orientation Test-Revised (Glaesmer et al., 2012) (LOT-R, a measure of optimism), and Connor Davidson Resilience Scale (Campbell-Sills and Stein, 2007) (CDRISC).

\section{Statistical Analysis}

Linear models were performed to examine the relationship between wisdom (the dependent variable) and gender (independent variable). Income, education, age, and marital status were also included as covariates because there were significant differences between women and men in this sample on those demographic areas. One model was calculated for 3D-WS total score, SD-WISE total score, and subscale scores for each measure. Cohen's $d$ was calculated for each gender effect. For the first set of exploratory analyses, a median split was calculated for 3D-WS total score, SD-WISE total score, and subscale scores for each measure. Separate linear models were performed to identify how the interaction between gender and the median split dummy coded variable impacted the relationship between gender and each wisdom variable. For the second set of exploratory analyses, separate linear models were performed to identify how the interaction between gender and each wisdom total score and subscale score impacted CES-D
(Depression), the UCLA-3 Loneliness, SF-36 Mental Well-being, LOT-R Optimism, and CD-RISC Resilience scores. These analyses were adjusted for family-wise error by using the false discovery rate (FDR) correction.

\section{RESULTS}

Detailed demographic comparison of men and women in the sample is presented in Table $\mathbf{1}$.

On the 3D-WS, the mean score among women was significantly higher on the Affective or Compassionate Dimension subscale score relative to men, $p=0.008$ with a medium effect size (Cohen's $d=0.481$ ). Men scored significantly higher on the Cognitive Dimension, $p=0.019$, with a small effect size (Cohen's $d=0.184$ ). Women also had higher 3D-WS total score, $p=0.01$, with a small effect size (Cohen's $d=0.292$ ). Please see Figure $1 \mathrm{~A}$ and Table 1.

There were significant differences between women and men in all six subscales of the SD-WISE (all $p<0.05$, Figure 1B and Table 1). These differences varied in direction such that men had higher scores on Emotional Regulation and Decisiveness, while women had higher scores on Insight, Tolerance of Divergent Values, Pro-social Behaviors, and Social Advising. These differences had small-to-medium effect sizes (Cohen's $d=0.110-0.331$ ), with the Emotion Regulation effect being the largest. There was no significant difference between women and men in the overall SD-WISE score, indicating that the relative strengths and weaknesses of each group balanced out in the overall score.

Median splits created "high wisdom" and "low wisdom" categories for each wisdom measure and subdomain to identify whether there was variability in size of gender effects by group. For 3D-WS Reflective Dimension, people in the "low wisdom" category had a larger gender difference such that women scored higher in wisdom than men, $p<0.001\left(\eta_{p}^{2}=0.010\right)$. There was no significant difference between women and men in the "high wisdom" group for 3D-WS Reflective Dimension, $p=0.17$. There were no other differences between "high wisdom" and "low wisdom" in any other categories.

Exploratory analyses examined how gender may moderate the relationship between wisdom and measures of well-being. Main effects supported the relationship between these variables and wisdom regardless of gender; however, gender did not moderate the relationship between wisdom and any of the measures of well-being including depression, loneliness, mental well-being, optimism, and resilience (Supplementary Tables 1-5).

\section{DISCUSSION}

This study on gender differences in wisdom found evidence that women and men differed on some components of wisdom. However, these differences were not uniform, but rather varied based on subdomains of wisdom as hypothesized. Women scored higher on several subdomains associated with social connection and compassion, including 3D-WS Affective 
TABLE 1 | Demographic and clinical characteristics of women and men.

\begin{tabular}{|c|c|c|c|c|c|c|c|c|c|}
\hline & \multicolumn{3}{|c|}{ Women } & \multirow[b]{2}{*}{ Range } & \multicolumn{3}{|c|}{ Men } & \multirow[b]{2}{*}{ Range } & \multirow[b]{2}{*}{$p$} \\
\hline & $N$ & Mean or \% & SD & & $N$ & Mean or \% & SD & & \\
\hline \multicolumn{10}{|l|}{ Sociodemographic } \\
\hline Age (years) & 334 & 64.76 & 19.8 & $27.35-103.78$ & 325 & 69.0 & 18.31 & $27.36-102.67$ & 0.004 \\
\hline Race (\% white) & 244 & 73.1 & & & 253 & 77.8 & & & 0.49 \\
\hline Education (\%) & & & & & & & & & 0.03 \\
\hline High School and Below & 34 & 10.18 & & & 25 & 7.7 & & & \\
\hline Some College to Bachelor's Degree & 181 & 54.19 & & & 162 & 49.85 & & & \\
\hline Post-Graduate Degree & 117 & 35.03 & & & 135 & 41.54 & & & \\
\hline Household Income (\%) & & & & & & & & & 0.001 \\
\hline$<\$ 35,000$ & 56 & 16.77 & & & 27 & 8.31 & & & \\
\hline$\$ 35,000-\$ 74,999$ & 61 & 18.26 & & & 59 & 18.15 & & & \\
\hline$\$ 75,000+$ & 160 & 47.90 & & & 190 & 58.46 & & & \\
\hline Current Marital Status (\% marriage-like) & 160 & 48.20 & & & 246 & 76.16 & & & $<0.001$ \\
\hline \multicolumn{10}{|l|}{ Wisdom Measures } \\
\hline 3-Dimensional Wisdom Scale (3D-WS) & 310 & 3.69 & 0.43 & & 314 & 3.56 & 0.45 & & 0.01 \\
\hline 3D-WS Cognitive Dimension & 310 & 3.55 & 0.50 & & 314 & 3.47 & 0.56 & & 0.019 \\
\hline 3D-WS Reflective Dimension & 310 & 3.91 & 0.55 & & 314 & 3.88 & 0.56 & & 0.94 \\
\hline 3D-WS Affective (Compassionate) Dimension & 310 & 3.60 & 0.51 & & 314 & 3.56 & 0.53 & & 0.008 \\
\hline San Diego Wisdom Scale (SD-WISE) & 310 & 3.86 & 0.43 & & 314 & 3.86 & 0.45 & & 0.55 \\
\hline SD-WISE Social Advising & 310 & 3.81 & 0.55 & & 314 & 3.71 & 0.62 & & 0.02 \\
\hline SD-WISE Decisiveness & 310 & 3.61 & 0.81 & & 314 & 3.83 & 0.70 & & 0.015 \\
\hline SD-WISE Emotional Regulation & 310 & 3.58 & 0.69 & & 314 & 3.80 & 0.64 & & $<0.001$ \\
\hline SD-WISE Self-Reflection & 310 & 3.85 & 0.62 & & 314 & 3.73 & 0.65 & & 0.03 \\
\hline SD-WISE Acceptance of Diverse Perspectives & 310 & 4.05 & 0.55 & & 314 & 3.89 & 0.62 & & 0.001 \\
\hline SD-WISE Pro-Social Behaviors & 310 & 4.28 & 0.49 & & & 4.23 & 0.50 & & 0.035 \\
\hline \multicolumn{10}{|l|}{ Wellbeing Measures } \\
\hline Depression (CES-D) & 319 & 5.56 & 5.27 & & 315 & 5.10 & 4.55 & & 0.24 \\
\hline Resilience (CD-RISC) & 329 & 29.94 & 6.09 & & 320 & 31.36 & 6.17 & & 0.03 \\
\hline Optimism (LOT-R) & 319 & 23.71 & 4.14 & & 317 & 23.71 & 3.96 & & 0.64 \\
\hline Loneliness (ULS) & 315 & 36.33 & 10.44 & & 314 & 36.61 & 10.18 & & 0.74 \\
\hline Mental Wellbeing (SF-36) & 326 & 52.90 & 9.21 & & 322 & 54.07 & 8.32 & & 0.09 \\
\hline
\end{tabular}

All Wisdom p-values corrected as described in sections "Materials and Methods" and "Results." CES-D, Center for Epidemiological Studies Depression Scale; CD-RISC, Connor-Davidson Resilience Scale; LOT-R, Life Orientation Task-Revised; ULS, UCLA Loneliness Scale. Bolded p values are significant; $p<0.05$.

or Compassionate Dimension, and SD-WISE Acceptance of Diverse Perspectives, Pro-Social Behavior and Social Advising subscales. We did not hypothesize differences in the reflection subdomains; women also scored higher on SD-WISE Insight subscale, and women in the lower half of the median split scored higher than men in the 3D-WS Reflective Dimension. On the other hand, men scored higher on SD-WISE and Emotional Regulation and Decisiveness subscales, and the Cognitive Dimension of the 3D-WS. Our third hypothesis was only confirmed by one of the wisdom measures: total wisdom scores were higher among women on the 3DWS but not on the SD-WISE total score. SD-WISE has 6 components and offers a more detailed examination of wisdom, with relative strengths in each gender being neutralized by relative weaknesses in others. Only one measure, the $3 \mathrm{D}$ WS Reflective, supported theory and evidence that there are larger gender differences among people with less wisdom. We also did not find any evidence that the gender moderated the relationship between wisdom and measures of well-being including depression, loneliness, mental well-being, optimism, and resilience.

Although some of these findings were unanticipated, others were well-aligned with past research in this and related areas. Acceptance of Diverse Perspectives and Social Advising both require perspective-taking and interest in the well-being and values of others even when they are misaligned with one's own. It seems likely that both of these domains are related to compassion, a domain that women reliably score higher (Eisenberg and Lennon, 1983; Sprecher and Fehr, 2005; Martins et al., 2013). Previous research has found evidence that older men score higher on cognitive domains of wisdom (Ardelt, 2009), and that older women score lower on these domains (Cheraghi et al., 2015). Cheraghi and colleagues' findings may have been significantly impacted by its setting in Iran, leading to much stronger differences than found in Ardelt's (2009) study or our own. In our study, we found that men of all ages scored higher on cognitive domains of wisdom and Decisiveness in particular, a novel finding. Our findings that men scored higher on Emotion 


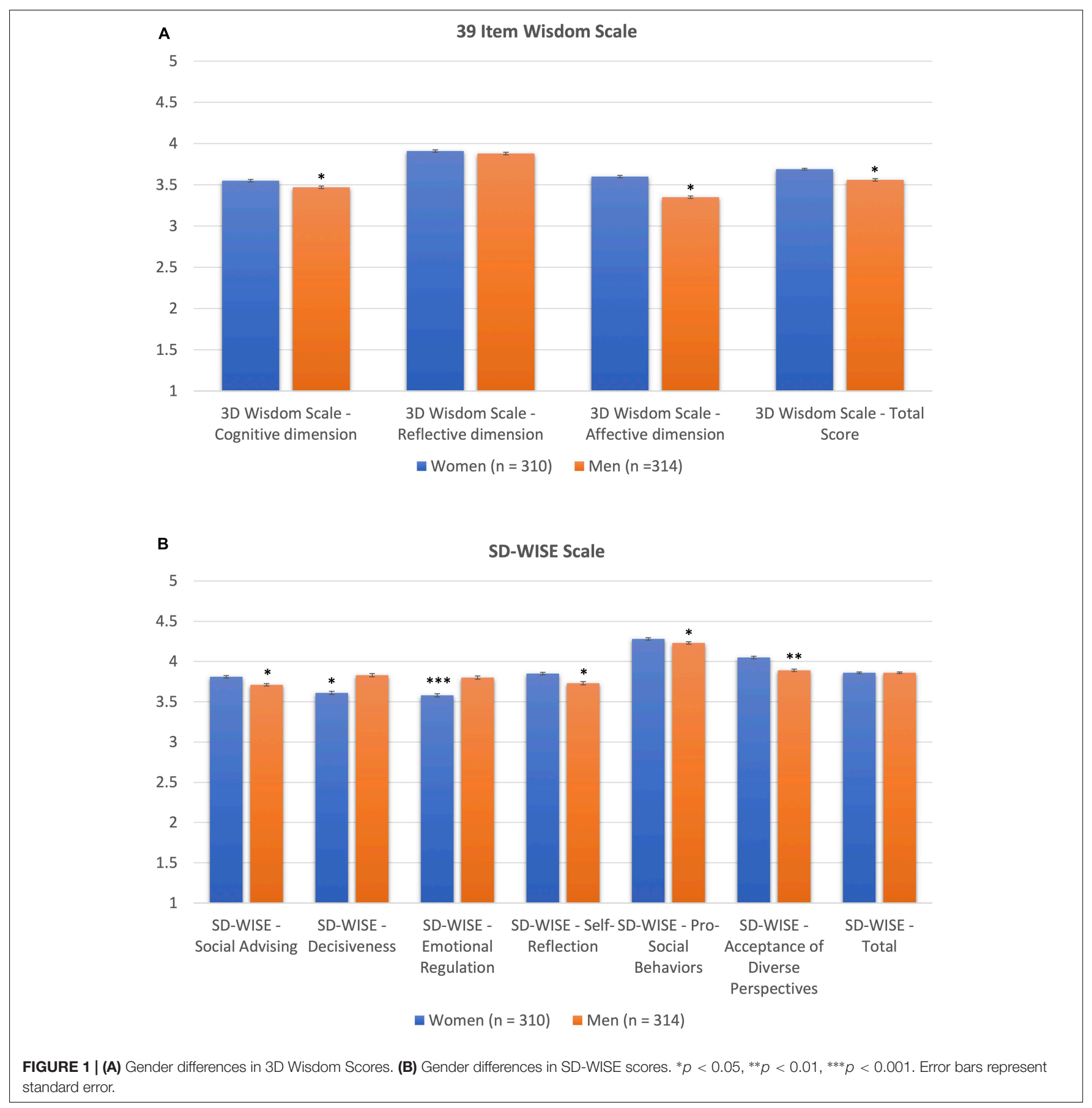

Regulation and women scored higher on one measure of SelfReflection were also novel.

There are two potential causes for the gender differences we identified. One is biological. In this regard, it is important not to conflate sex and gender, but rather to discuss the potential impact of biological processes including sex on differences in wisdom. The finding of greater empathy and compassion toward others in women has been reported across time periods and across cultures. Sex-based differences in oxytocin receptor gene polymorphism may lead to increased empathy in women
(Wu et al., 2012). In a different lab-based study, women and men performed similarly on three empathy tasks, but women activated more emotion- and self-based regions of the brain while men activated more cognitive-based regions, somewhat aligned with our findings (Derntl et al., 2010). In another study, women and men showed differences in neural activity during completion of emotion regulation tasks, although it was unclear whether these differences were innate and/or learned (McRae et al., 2008). This latter concept is important to consider; variance in biological processes can be externally caused, driven 
by sociocultural processes and the socialization that results. These findings, taken together, might indicate that individuals may use different strategies to achieve wisdom-associated goals based on biological and/or sociocultural processes, but that the strategy itself may not be particularly important as long as it works well for the individual and taps into at least one wisdom subcomponent. That is, it may not be necessary to have a high level of wisdom in all areas as long as the strengths an individual does have work effectively across aspects of that person's life.

These sociocultural processes mentioned are the second, and likely to be the most influential cause for the observed gender difference in wisdom, including social expectations, gender norms and how wisdom-relevant behaviors are differentially reinforced between boys/men and girls/women by parents, teachers, peers, and society at large. Boys and men tend to be socialized toward behaviors including toughness and leadership, which may translate into being more decisive and in control of emotions, whereas girls and women tend to be socialized toward behaviors including warmth and caretaking, which may translate into pro-social behaviors including being compassionate and accepting of diverse people and ideas (Eagly, 2013). Male adolescents conceptualize wise people as somewhat more calculating, strict, and questioning than female adolescents do (Hollingworth et al., 2013); a description that fits well with being decisive and more focused on cognitive processes rather than emotion. Female adolescents conceptualize wise people as somewhat more cooperative, optimistic, extroverted, and spontaneous than male adolescents do (Hollingworth et al., 2013); which similarly aligns with being compassionate and oriented toward others. Additionally, men tend to report that work situations help develop wisdom, while women report that family-related and loss situations help develop wisdom (Glück et al., 2009). Furthermore, when people are asked to describe a wise man or a wise woman, wise women are somewhat more likely to be described as having compassion for others (Glück et al., 2009). Therefore, it appears that there are differences in how people conceptualize and actively develop wisdom by gender, indicating sociocultural causes based in gender norms. This may similarly indicate that women and men focus on different subdomains of wisdom as personal areas of growth to work toward, based on what they personally value, which is naturally situated within sociocultural context and gender norms. Of course, there is variance in gender norms by culture and region, and these norms change over time and by generation. This may explain why there is some variability in findings about gender differences in wisdom, given that research has been conducted in multiple different countries and regions with samples of different racial groups and age groups (e.g., Yang, 2001; Kanwar, 2013; Bang, 2015; Cheraghi et al., 2015; Maroof et al., 2015).

It has been argued that wisdom should be a broadly gender-neutral construct (Levenson, 2009) so that it does not facilitate gender bias by promoting attributes that current gender norms associate with one gender or another. Another theory (Orwoll and Achenbaum, 1993; Ardelt, 2009) posited that gender differences in wisdom may only be present at lower levels of wisdom, because very wise people will be strong in all wisdom subdomains. However, we found evidence for this in only one subdomain of wisdom, 3D-WS Reflective. Given our findings, a two-prong question can be considered: is the construct of wisdom gender neutral, and if it is, are we observing gender bias in current measures of wisdom? Our first comment regarding this question is that the route to wisdom does not have to be identical for each person- and indeed, we would argue that a society benefits from diversity in this respect, as in many other respects. Additionally, as observed in the emotion regulation and empathy literature (e.g., McRae et al., 2008; Derntl et al., 2010), individuals may use different strategies or subdomains of wisdom to achieve similar goals, indicating that there are many paths to a wise life, and high levels of wisdom in each subdomain is not necessary. For example, a woman and a man pursuing wisdom through individualized pathways might react to interpersonal conflict in different ways. The woman, with strengths in compassion, selfreflection, and acceptance of diverse perspectives, might seek to understand the conflict through the other person's point of view, and show compassion for that person and their perspective even if they disagree. In contrast, the man, with strengths in emotional regulation and decisiveness, might retain emotional equilibrium despite the conflict and come to an understanding within himself that their relationship with that other person is more important than the issue they are disagreeing about, and therefore, decide to move beyond it instead of continuing the conflict. Therefore, the outcome may look similar from the outside - prioritizing personal relationship above the conflict -but the strategies used to achieve the outcome would be different.

Therefore, it seems to us that variance by subgroup in strengths and weaknesses at the wisdom subdomain level is not a flaw. It may not indicate gender bias as much as it does allowing for a diversity of paths toward wisdom. However, given the alignment between our findings and what has been previously noted to be valued as wise behavior by women and men, we wonder whether these differences might lessen as the divide in societal expectations of women and men fades. We would also note that these effects were small, indicating that although differences existed, women and men still had meaningful overlap as groups in their wisdom scores.

Finally, it seems that, at the macro level, the SD-WISE measure does not show evidence of an overall gender difference, unlike 3D-WS. The subcomponents included in a measure of wisdom will of course impact gender and other group differences. These two measures of wisdom measures were developed conceptually and tested psychometrically, using theoretical and empirical findings on layperson and expert definitions of wisdom without focusing on gender balance (c.f., Ardelt, 2003; Thomas et al., 2019; Jeste et al., 2020b,c). Variance in development choices (for example, separating out aspects of cognitive subcomponents of wisdom in the SD-WISE compared in combining them into one scale in the 3D-WS) may have influenced balance and therefore our findings. Given that wisdom is intended to be balanced, or without bias at least in its total score, measure developers, and researchers choosing measures, should consider these issues to avoid accidental bias. Glück et al.'s (2013) paper assessed five measures of wisdom, including the 3D-WS (the SD-WISE 
was developed later) and provided general recommendations to guide selection.

\section{Study Limitations}

The cross-sectional design prevents assessment of differences in how wisdom develops and evolves over the lifespan. Future longitudinal work will be able to fully describe this development by gender, and the influence of other important factors including those associated with mental health and wellbeing. Longitudinal work may also be able to examine some of the hypotheses we and others have considered in regards to why and how gender differences occur. Participants self-identified their genders, and only binary options were offered so there were no options for people who are non-binary or other genders. Understanding wisdom profiles of non-binary people in future studies would be particularly enlightening in understanding how wisdom develops among people who may be less bound to traditional gender norms. We did not collect data to identify how many participants were transgender, which would aid in understanding whether transgender women and men have unique wisdom profiles relative to cis women and men. We did not collect any biomarkers relevant to gender or sex, so assessment of the potential impact of hormones or other factors was not possible. The study sample was predominantly white and came from an urban county in the United States; thus, the findings may not apply to other race/ethnic groups and different cultural regions, and study of other groups is important. We should point out, however, that in a recent study using SD-WISE and ULS scale for loneliness, we found that the constructs of wisdom and loneliness seemed to be largely similar in a San Diego sample of middle-aged and older adults and an age-comparable sample from rural Italy (Jeste et al., 2020a).

\section{Implications}

On average, both women and men have strengths in wisdom subdomains that can be capitalized upon to promote their wellbeing. Helping people identify and lean on these strengths may promote related aspects of well-being including social connection and happiness. We also find that both groups have relative weaknesses that may benefit from individual and societal intervention to improve well-being and promote healthy living, including the growing set of positive psychiatry interventions. Consistent with past literature, we find a difference in compassion between women and men. There are a number of compassion interventions including compassion-focused therapy (Kirby et al., 2017). We also found that women scored higher in self-reflection than men. Engagement in most therapies promotes self-reflection and insight, making it a useful approach for those looking to strengthen these subdomains. Additionally, spiritual and non-therapy-based mindfulness practices may also promote insight. We found that men are higher in decisiveness than women. It is worth adding that the SD-WISE is unique in being validated as a wisdom scale with decisiveness as one of its major components. Intervention strategies like problem-solving therapy (Bell and D'Zurilla, 2009) may improve decisiveness and well-being. Similarly, this study is the first to report that men are higher in emotional regulation than women; mindfulness-based interventions including mindfulness-based stress reduction can improve emotion regulation (Guendelman et al., 2017). The finding of gender differences in components of wisdom suggests that assessing relative strengths and weaknesses in wisdom subdomains at the individual level may be helpful in guiding treatment planning across groups. However, our results did not find evidence that these varying wisdom profiles between women and men impacted the relationship between wisdom and other constructs typically targeted by positive psychiatry interventions, like resilience and optimism. One possible implication is that tailoring positive psychiatry intervention to individual wisdom profiles may be an effective strategy to improving these other, consistently associated constructs. Public health initiatives that target key subdomains among subgroups may be helpful to promote wellness.

\section{DATA AVAILABILITY STATEMENT}

The raw data supporting the conclusions of this article will be made available by the authors, without undue reservation.

\section{ETHICS STATEMENT}

The studies involving human participants were reviewed and approved by the University of California, San Diego Human Research Protections Program. Written informed consent for participation was not required for this study in accordance with the national legislation and the institutional requirements.

\section{AUTHOR CONTRIBUTIONS}

ET, BP, EL, and DJ conceptualized the study. ET completed the initial analyses with conceptual support from DJ and conceptual and pragmatic support from T-CW, MT, and XT, wrote the first draft of the manuscript, and made the first draft of the tables and figures. $\mathrm{RD}$ provided database management and analytic support. All authors contributed to revisions of the manuscript, tables, and figures.

\section{FUNDING}

This work was supported in part by the Veterans Affairs Rehabilitation Research and Development grant 5IK2RX00307902 (PI: ET), NIMH R01 MH120201 (PI: BP), NIMH K23MH119375-01 (PI: EL), and Stein Institute for Research on Aging (Director: DJ). This study does not represent the views of the United States Federal Government.

\section{SUPPLEMENTARY MATERIAL}

The Supplementary Material for this article can be found online at: https://www.frontiersin.org/articles/10.3389/fpsyg.2021. 769294/full\#supplementary-material 


\section{REFERENCES}

Aldwin, C. M. (2009). Gender and wisdom: a brief overview. Res. Hum. Dev. 6, 1-8. doi: 10.1080/15427600902779347

Alexander, D., and Andersen, K. (1993). Gender as a factor in the attribution of leadership traits. Polit. Res. Q. 46, 527-545. doi: 10.1177/106591299304600305

Ardelt, M. (1997). Wisdom and life satisfaction in old age. J. Gerontol. Ser. B Psychol. Sci. Soc. Sci. 52B, 15-27. doi: 10.1093/geronb/52B.1.P15

Ardelt, M. (2003). Empirical Assessment of a Three-Dimensional Wisdom Scale. Res. Aging 25, 275-324. doi: 10.1177/0164027503025003004

Ardelt, M. (2009). How Similar are Wise Men and Women? A Comparison Across Two Age Cohorts. Res. Hum. Dev. 6, 9-26. doi: 10.1080/15427600902779354

Bang, H. (2015). African American Undergraduate Students' Wisdom and EgoIdentity Development: effects of Age, Gender, Self-Esteem, and Resilience. J. Black Psychol. 41, 95-120. doi: 10.1177/0095798413510176

Bang, H., and Zhou, Y. (2014). The function of wisdom dimensions in ego-identity development among Chinese university students. Int. J. Psychol. 49, 434-445. doi: 10.1002/ijop.12065

Bangen, K. J., Meeks, T. W., and Jeste, D. V. (2013). Defining and Assessing Wisdom: a Review of the Literature. Am. J. Geriatr. Psychiatry 21, 1254-1266. doi: 10.1016/j.jagp.2012.11.020

Bell, A. C., and D'Zurilla, T. J. (2009). Problem-solving therapy for depression: a meta-analysis. Clin. Psychol. Rev. 29, 348-353. doi: 10.1016/j.cpr.2009.02.003

Booker, J. A., and Dunsmore, J. C. (2016). Profiles of wisdom among emerging adults: associations with empathy, gratitude, and forgiveness. J. Posit. Psychol. 11, 315-325. doi: 10.1080/17439760.2015.1081970

Brienza, J. P., Kung, F. Y. H., Santos, H. C., Bobocel, D. R., and Grossmann, I. (2018). Wisdom, bias, and balance: toward a process-sensitive measurement of wisdom-related cognition. J. Pers. Soc. Psychol. 115, 1093-1126.

Campbell-Sills, L., and Stein, M. B. (2007). Psychometric analysis and refinement of the connor-davidson resilience scale (CD-RISC): validation of a 10-item measure of resilience. J. Trauma. Stress 20, 1019-1028. doi: 10.1002/jts.20271

Cheraghi, F., Kadivar, P., Ardelt, M., Asgari, A., and Farzad, V. (2015). Gender as a Moderator of the Relation Between Age Cohort and Three-Dimensional Wisdom in Iranian Culture. Int. J. Aging Hum. Dev. 81, 3-26. doi: 10.1177/ 0091415015616394

Dahlsgaard, K., Peterson, C., and Seligman, M. E. P. (2005). Shared Virtue: the Convergence of Valued Human Strengths across Culture and History. Rev. Gen. Psychol. 9, 203-213. doi: 10.1037/1089-2680.9.3.203

Derntl, B., Finkelmeyer, A., Eickhoff, S., Kellermann, T., Falkenberg, D. I., Schneider, F., et al. (2010). Multidimensional assessment of empathic abilities: neural correlates and gender differences. Psychoneuroendocrinology 35, 67-82. doi: 10.1016/j.psyneuen.2009.10.006

Eagly, A. H. (2013). Sex Differences in Social Behavior: A Social-role interpretation. East Sussex: Psychology Press.

Eisenberg, N., and Lennon, R. (1983). Sex Differences in Empathy and Related Capacities. Psychol. Bull. 94, 100-131. doi: 10.1037/0033-2909.94.1.100

Glaesmer, H., Rief, W., Martin, A., Mewes, R., Brähler, E., Zenger, M., et al. (2012). Psychometric properties and population-based norms of the Life Orientation Test Revised (LOT-R). Br. J. Health Psychol. 17, 432-445. doi: 10.1111/j.20448287.2011.02046.x

Glück, J. (2020). The Important Difference Between Psychologists' Labs and Real Life: evaluating the Validity of Models of Wisdom. Psychol. Inq. 31, 144-150. doi: 10.1080/1047840X.2020.1750909

Glück, J., and Bluck, S. (2011). Laypeople's conceptions of wisdom and its development: cognitive and integrative views. J. Gerontol. B Psychol. Sci. Soc. Sci. 66, 321-324. doi: 10.1093/geronb/gbr011

Glück, J., König, S., Naschenweng, K., Redzanowski, U., Dorner, L., Straßer, I., et al. (2013). How to measure wisdom: content, reliability, and validity of five measures. Front. Psychol. 4:405. doi: 10.3389/fpsyg.2013.00405

Glück, J., Strasser, I., and Bluck, S. (2009). Gender Differences in Implicit Theories of Wisdom. Res. Hum. Dev. 6, 27-44. doi: 10.1080/15427600902779370

Guendelman, S., Medeiros, S., and Rampes, H. (2017). Mindfulness and Emotion Regulation: insights from Neurobiological, Psychological, and Clinical Studies. Front. Psychol. 8:220. doi: 10.3389/fpsyg.2017.00220

Heintz, S., Kramm, C., and Ruch, W. (2019). A meta-analysis of gender differences in character strengths and age, nation, and measure as moderators. J. Posit. Psychol. 14, 103-112. doi: 10.1080/17439760.2017.1414297
Hines, M. (2020). Neuroscience and Sex/Gender: looking Back and Forward. J. Neurosci. 40, 37-43. doi: 10.1523/JNEUROSCI.0750-19.2019

Hofstede, G. (1996). Gender stereotypes and partner preferences of Asian women in masculine and feminine cultures. J. Cross Cult. Psychol. 27, 533-546. doi: $10.1177 / 0022022196275003$

Hollingworth, L., Sánchez-Escobedo, P., Graudina, L., Misiuniene, J., and Park, K. (2013). Gender Differences on the Concept of Wisdom: an International Comparison. Gift. Talent. Int. 28, 219-225. doi: 10.1080/15332276.2013. 11678416

Hyde, J. S., Bigler, R. S., Joel, D., Tate, C. C., and van Anders, S. M. (2019). The future of sex and gender in psychology: five challenges to the gender binary. Am. Psychol. 74, 171-193. doi: 10.1037/amp0000307

Jeste, D. V., Lee, E. E., Palmer, B. W., and Treichler, E. B. H. (2020b). Moving from Humanities to Sciences: a New Model of Wisdom Fortified by Sciences of Neurobiology. Med. Evol. Psychol. Inq. 31, 134-143. doi: 10.1080/1047840X. 2020.1757984

Jeste, D. V., Thomas, M. L., Liu, J., Daly, R. E., Tu, X. M., Treichler, E. B. H., et al. (2020c). Is Spirituality a Component of Wisdom? Study of 1,786 Adults Using Expanded San Diego Wisdom Scale (Jeste-Thomas Wisdom Index). J. Psychiatr. Res. 132, 174-181. doi: 10.1016/j.jpsychires.2020.09.033

Jeste, D. V., Di Somma, S., Lee, E. E., Nguyen, T. T., Scalcione, M., Biaggi, A., et al. (2020a). Study of loneliness and wisdom in 482 middle-aged and oldestold adults: a comparison between people in Cilento, Italy and San Diego, USA. Aging Ment. Health 25, 2149-2159. doi: 10.1080/13607863.2020.1821170

Jeste, D. V., Palmer, B. W., Rettew, D. C., and Boardman, S. (2015). Positive Psychiatry: its Time Has Come. J. Clin. Psychiatry 76, 675-683. doi: 10.4088/ JCP.14nr09599

Kanwar, M. (2013). Exploring wisdom in relationship with coping styles, social desirability, subjective wellbeing, age and gender. Indian J. Posit. Psychol. 4, 227-234.

Kim, K. R., Song, Y. Y., Park, J. Y., Lee, E. H., Lee, M., Lee, S. Y., et al. (2013). The relationship between psychosocial functioning and resilience and negative symptoms in individuals at ultra-high risk for psychosis. Aust. N. Z. J. Psychiatry 47, 762-771. doi: 10.1177/0004867413488218

Kirby, J. N., Tellegen, C. L., and Steindl, S. R. (2017). A Meta-Analysis of Compassion-Based Interventions: current State of Knowledge and Future Directions. Behav. Ther. 48, 778-792. doi: 10.1016/j.beth.2017.06.003

Kiyar, M., Collet, S., T'Sjoen, G., and Mueller, S. C. (2020). Neuroscience in transgender people: an update. Neuroforum 26, 85-92. doi: 10.1515/nf-20200007

König, S., and Glück, J. (2013). Individual Differences in Wisdom Conceptions: relationships to Gratitude and Wisdom. Int. J. Aging Hum. Dev. 77, 127-147. doi: 10.2190/AG.77.2.c

König, S., and Glück, J. (2014). "Gratitude Is With Me All the Time”: how gratitude relates to wisdom. J. Gerontol. B Psychol. Sci. Soc. Sci. 69, 655-666. doi: 10.1093/ geronb/gbt123

Lee, E. E., Bangen, K. J., Avanzino, J. A., Hou, B., Ramsey, M., Eglit, G., et al. (2020). Outcomes of Randomized Clinical Trials of Interventions to Enhance Social, Emotional, and Spiritual Components of Wisdom: a Systematic Review and Meta-analysis. JAMA Psychiatry 77:925. doi: 10.1001/jamapsychiatry.2020.0821

Levenson, M. R. (2009). Gender and Wisdom: the Roles of Compassion and Moral Development. Res. Hum. Dev. 6, 45-59. doi: 10.1080/15427600902782127

Levenson, M. R., Jennings, P. A., Aldwin, C. M., and Shiraishi, R. W. (2005). SelfTranscendence: conceptualization and Measurement. Int. J. Aging Hum. Dev. 60, 127-143. doi: 10.2190/XRXM-FYRA-7U0X-GRC0

Maroof, R., Khan, M. J. Z., Anwar, M., and Anwar, A. (2015). A Cross-Sectional Study of Wisdom: a Matter of Age and Gender. FWU J. Soc. Sci. 9, 63-71.

Martins, D., Nicholas, N. A., Shaheen, M., Jones, L., and Norris, K. (2013). The Development and Evaluation of a Compassion Scale. J. Health Care Poor Underserved 24, 1235-1246. doi: 10.1353/hpu.2013.0148

McLaughlin, P. T., McMinn, M. R., Morse, M., Neff, M. A., Johnson, B., Summerer, D., et al. (2018). The effects of a wisdom intervention in a Christian congregation. J. Posit. Psychol. 13, 502-511. doi: 10.1080/17439760. 2017.1350739

McRae, K., Ochsner, K. N., Mauss, I. B., Gabrieli, J. J. D., and Gross, J. J. (2008). Gender Differences in Emotion Regulation: an fMRI Study of Cognitive Reappraisal. Group Process. Intergroup Relat. 11, 143-162. doi: 10.1177/ 1368430207088035 
Meeks, T. W., and Jeste, D. V. (2009). Neurobiology of wisdom: a literature overview. Arch. Gen. Psychiatry 66:355. doi: 10.1001/archgenpsychiatry.2009.8

Nolen-Hoeksema, S. (2012). Emotion regulation and psychopathology: the role of gender. Annu. Rev. Clin. Psychol. 8, 161-187. doi: 10.1146/annurev-clinpsy032511- 143109

Nolen-Hoeksema, S., and Aldao, A. (2011). Gender and age differences in emotion regulation strategies and their relationship to depressive symptoms. Pers. Individ. Differ. 51, 704-708. doi: 10.1016/j.paid.2011.06.012

Orwoll, L., and Achenbaum, A. (1993). Gender and the development of wisdom. Hum. Dev. 36, 274-296. doi: 10.1159/000278214

Paredes, A. M., Lee, E. E., Chik, L., Gupta, S., Palmer, B. W., Palinkas, L. A., et al. (2020). Qualitative study of loneliness in a senior housing community: the importance of wisdom and other coping strategies. Aging Ment. Health 25, 559-566. doi: 10.1080/13607863.2019.1699022

Peterson, C., and Seligman, M. E. P. (2004). Character Strengths and Virtues: A Handbook and Classification. Washington: American Psychological Association.

Pommier, E., Neff, K. D., and Tóth-Király, I. (2020). The Development and Validation of the Compassion Scale. Assessment 27, 21-39. doi: 10.1177/ 1073191119874108

Radloff, L. S. (1977). The CES-D Scale: a Self-Report Depression Scale for Research in the General Population. Appl. Psychol. Meas. 1, 385-401. doi: 10.1177/ 014662167700100306

Rametti, G., Carrillo, B., Gómez-Gil, E., Junque, C., Segovia, S., Gomez, Á, et al. (2011). White matter microstructure in female to male transsexuals before cross-sex hormonal treatment. A diffusion tensor imaging study. J. Psychiatr. Res. 45, 199-204. doi: 10.1016/j.jpsychires.2010.05.006

Richards, C., Bouman, W. P., Seal, L., Barker, M. J., Nieder, T. O., and T'Sjoen, G. (2016). Non-binary or genderqueer genders. Int. Rev. Psychiatry 28, 95-102. doi: 10.3109/09540261.2015.1106446

Ronk, L. L. (1993). Gender gaps within management. Nurs. Manag. 24:65.

Russell, D. W. (1996). UCLA Loneliness Scale (Version 3): reliability, Validity, and Factor Structure. J. Pers. Assess. 66, 20-40. doi: 10.1207/s15327752jpa6601_2

Singh, U., and Dahiya, S. (2013). Gender differences in wisdom among school students. Indian J. Health Wellbeing 4, 1573-1576.

Sprecher, S., and Fehr, B. (2005). Compassionate love for close others and humanity. J. Soc. Pers. Relat. 22, 629-651. doi: 10.1177/0265407505056439

Staudinger, U. M., and Glück, J. (2011). Psychological Wisdom Research: commonalities and Differences in a Growing Field. Annu. Rev. Psychol. 62, 215-241. doi: 10.1146/annurev.psych.121208.131659

Thomas, M. L., Bangen, K. J., Palmer, B. W., Martin, A. S., Avanzino, J. A., Depp, C. A., et al. (2019). A new scale for assessing wisdom based on common domains and a neurobiological model: the San Diego Wisdom Scale (SDWISE). J. Psychiatr. Res. 108, 40-47. doi: 10.1016/j.jpsychires.2017.09.005
Thomas, M. L., Kaufmann, C. N., Palmer, B. W., Depp, C. A., Martin, A. S., Glorioso, D. K., et al. (2016). Paradoxical Trend for Improvement in Mental Health with Aging: a Community-Based Study of 1,546 Adults Aged 21-100 Years. J. Clin. Psychiatry 77, e1019-e1025. doi: 10.4088/JCP.16m 10671

Treichler, E. B. H., Glorioso, D., Lee, E. E., Wu, T.-C., Tu, X. M., Daly, R., et al. (2020). A pragmatic trial of a group intervention in senior housing communities to increase resilience. Int. Psychogeriatr. 32, 173-182. doi: 10. $1017 /$ S1041610219002096

Ware, J. E. J. (2000). SF-36 Health Survey Update. Spine 25, 3130-3139. doi: 10.1097/00007632-200012150-00008

Webster, J. D. (2003). An exploratory analysis of a self-assessed Wisdom Scale. J. Adult Dev. 10, 13-22.

Webster, J. D., Bohlmeijer, E. T., and Westerhof, G. J. (2014). Time to flourish: the relationship of temporal perspective to well-being and wisdom across adulthood. Aging Ment. Health 18, 1046-1056. doi: 10.1080/13607863.2014. 908458

$\mathrm{Wu}, \mathrm{N} ., \mathrm{Li}, \mathrm{Z}$., and Su, Y. (2012). The association between oxytocin receptor gene polymorphism (OXTR) and trait empathy. J. Affect. Disord. 138, 468-472. doi: 10.1016/j.jad.2012.01.009

Yang, S. (2001). Conceptions of Wisdom among Taiwanese Chinese. J. Cross Cult. Psychol. 32, 662-289.

Zimmermann, P., and Iwanski, A. (2014). Emotion regulation from early adolescence to emerging adulthood and middle adulthood: age differences, gender differences, and emotion-specific developmental variations. Int. J. Behav. Dev. 38, 182-194. doi: 10.1177/0165025413515405

Conflict of Interest: The authors declare that the research was conducted in the absence of any commercial or financial relationships that could be construed as a potential conflict of interest.

Publisher's Note: All claims expressed in this article are solely those of the authors and do not necessarily represent those of their affiliated organizations, or those of the publisher, the editors and the reviewers. Any product that may be evaluated in this article, or claim that may be made by its manufacturer, is not guaranteed or endorsed by the publisher.

Copyright (c) 2022 Treichler, Palmer, Wu, Thomas, Tu, Daly, Lee and Jeste. This is an open-access article distributed under the terms of the Creative Commons Attribution License (CC BY). The use, distribution or reproduction in other forums is permitted, provided the original author(s) and the copyright owner(s) are credited and that the original publication in this journal is cited, in accordance with accepted academic practice. No use, distribution or reproduction is permitted which does not comply with these terms. 